\title{
ACTIVIDADES EMPRENDIDAS PARA RECUPERAR Y PRESERVAR LA RAZA OVINA CHURRA TENSINA
}

\author{
UNDERTAKEN ACTIVITIES TO RECOVER AND PRESERVE THE \\ CHURRA TENSINA SHEEP BREED
}

Sanz, A. ${ }^{1 *}$, Calvo, J.H. ${ }^{2}$, Martí. J.I. ${ }^{1}$, Alabart, J.L. ${ }^{1}$, Joy, M. ${ }^{1}$, Ripoll, G. ${ }^{1}$ y Álvarez-Rodríguez, J. ${ }^{1}$

${ }^{1}$ Centro de Investigación y Tecnología Agroalimentaria de Aragón (CITA). Avda. Montañana 930. 50059

Zaragoza. España. *asanz@aragon.es

${ }^{2}$ Fundación ARAID. Zaragoza. España.

\section{PaLABRAS CLAVE ADICIONALES}

Razas amenazadas. Ovino. Churro. Germoplasma. Genética.

\section{RESUMEN}

En el caso de la raza Churra Tensina, en peligro de extinción y originaria del Pirineo Oscense, se realizó una prospección de individuos y una caracterización del sistema productivo a través de encuestas a ganaderos, definiéndose explotaciones de mediano tamaño que tienen su continuidad garantizada. Se estudió la morfología racial a partir de medidas zoométricas y caracteres fanerópticos de los animales inscritos en la Asociación (ATURA), lo que permitió clasificar la raza como mediolínea, dolicocéfala y eumétrica. Se estudiaron los parámetros productivos y reproductivos, así como la calidad de canal y carne de los tipos de cordero lechal, ternasco y pastenco, confirmando su viabilidad comercial. La caracterización genética mostró una raza homogénea, con niveles altos de variabilidad genética y bajos de consanguinidad. Finalmente, se han creado bancos de semen y embriones, estando representados los distintos genotipos de resistencia a Scrapie. La evolución positiva del censo en los últimos 10 años, añadida a la viabilidad de sus productos comerciales, garantiza la preservación de la Churra Tensina y de determinados sistemas ganaderos ligados a la tierra.

\section{SUMMARY}

This work aims to present the research activities in the Churra Tensina sheep breed,

$\overline{\text { Presentado al }}$ Congreso SERGA (2010, Asturias).

\section{AdDITIONAL KEYWORDS}

Endangered breeds. Sheep. Churro. Germplasm. Genetics.

raised on the mountain area of Huesca Pyrenees. The population structure was prospected and the farming system was characterized through surveys to farmers. The animal morphology was studied through zoometric measurements and phaneroptic characters assessment of the individuals belonging to the breeders' Association (ATURA), showing a mediolineous, dolicocephalous and eumetric breed. The productive and reproductive parameters were analysed, and the commercial value of the suckling, light and heavy lamb categories was confirmed. The genetic studies showed that Churra Tensina is an homogeneous breed, with high genetic variability and low inbreeding. This breed was self-genetic differentiated, and has no crossbreeding with other Churro group populations. Finally, semen and embryos banks were established, being the different Scrapie resistance genotypes represented. The increasing census evolution in the past 10 years and the observed commercial viability assure the long term maintenance of this breed in their original location.

\section{INTRODUCCIÓN}

La raza Churra Tensina debe su denominación al hecho de asentarse ancestralmente en el valle de Tena del Pirineo Central español. Esta raza fue apreciada por su capacidad de aprovechar pastos difíciles y sopor- 
tar condiciones climáticas adversas. Sin embargo, estas ventajas no consiguieron frenar la caída en su número de efectivos, considerándose en peligro de extinción (BOE, 1997), y según el criterio de la FAO (1996) como una raza en riesgo-mantenida. En este trabajo se presenta un resumen de las actividades emprendidas en el CITA de Aragón con el fin de recuperar y preservar la raza.

\section{MATERIAL YMÉTODOS}

Para conocer la caracterización estructural de la raza, en 2006, se realizaron encuestas personalizadas en las 15 explotaciones pertenecientes a la Asociación de Criadores (ATURA). Con objeto de describir la morfología de la raza, se estudiaron 15 variables zoométricas y los índices corporales en 85 animales adultos, 53 hembras vacías y 32 machos, procedentes de 4 explotaciones (Álvarez-Rodríguez et al., 2008).

Se realizó la caracterización reproductiva de la raza en 32 corderas, a las que se tomaron muestras semanales de sangre para determinar niveles de progesterona entre los 6 y los 14,5 meses de edad (Sanz y Álvarez-Rodríguez, 2008). También se determinó la duración del anestro post-parto en 48 ovejas con parto en primavera (fecha media: 25 de marzo), considerando los niveles semanales de progesterona observados del parto al último día de primavera.

Además, se estudió la posibilidad de diversificación de la producción ovina en Aragón, a través de la comparación de la calidad de la canal de distintos tipos comerciales de cordero de raza Churra Tensina. Se estudiaron las canales procedentes de 42 corderos machos de parto simple, sacrificados a 9-12 kg (lechal), 20-24 kg (ternasco) y 28-35 kg de peso vivo (pastenco), según se describe por Sanz et al. (2008).

Para estimar la variabilidad genética en la raza Churra Tensina y detectar la posible existencia de un flujo genético con otras razas del tronco Churro explotadas en Espa- ña se analizaron 28 microsatélites de ADN recomendados por la FAO en 309 animales pertenecientes a las razas Churra Tensina $(n=65)$, Churra Lebrijana $(n=65)$, Churra $(n=$ $60)$, Latxa $(n=51)$, Merino $(n=29)$ y Muflón $(\mathrm{n}=39)$ (Calvo et al., 2011).

\section{RESULTADOSYDISCUSIÓN}

\section{CaRACTERIZACIÓN DEL SISTEMADE EXPLO- TACIÓN}

Las explotaciones se encuentran localizadas en su mayoría en las comarcas del Sobrarbe (40\%), Alto Gállego (20\%), Jacetania (13\%) y Ribagorza (7\%), en el Pirineo Oscense, y en puntos aislados de la geografía aragonesa. El tamaño medio de los rebaños es de 256 ovejas adultas (de ellas, 182 Churra Tensina), con un ratio de 31 reproductoras por morueco y 34 corderas de reposición por explotación. El tamaño de la base animal es sensiblemente menor al encontrado en otros grupos raciales en zonas de montaña adyacentes (Avellanet et $a l ., 2005)$. El origen de las corderas y de los moruecos de reposición es exclusivamente propio en un $87 \%$ y un $67 \%$ de los casos, respectivamente; no obstante, se ha comprobado un buen grado de diversidad genética en esta raza.

Un 93\% de las explotaciones producen mayoritariamente corderos ligeros tipo Ternasco (20-25 $\mathrm{kg}$ peso). En los últimos 10 años un $73 \%$ de las explotaciones han crecido de tamaño, tendencia acorde con la evolución del censo de animales inscritos en el Libro Genealógico, que ha pasado de 837 individuos en el año 2000 a 8500 en el año 2010. La mayoría de los ganaderos (80\%) aspiran a mantener la actividad en los próximos años.

\section{ESTUDIO MORFO-ESTRUCTURAL YZOOTÉC- NICO DE LA RAZA}

La talla media de la raza, definida por su alzada a la cruz y alzada a la grupa, fue el carácter más uniforme. El peso vivo y la 


\section{CONSERVACIÓN DE LA RAZA OVINA CHURRA TENSINA}

mayoría de las medidas cefálicas y del tronco fueron inferiores a los recogidos en la base de datos de la FAO en las razas filogenéticamente próximas Churra y Churra Lebrijana (FAO, 1996), que podría ser resultado de su explotación en ambientes duros de montaña que han implicado normalmente fuertes desplazamientos verticales durante el pastoreo.

El índice corporal indicó para ambos sexos la forma mediolínea. El índice cefálico clasificó la raza como marcadamente dolicocéfala, ya que predomina la longitud de la cabeza en relación a su anchura. El índice pelviano permitió clasificar la grupa de esta raza como convexilínea. La mayoría de los índices funcionales mostró un marcado dimorfismo sexual y una ausencia de efecto de la edad. Los resultados del índice dáctilo-torácico indican que la raza es eumétrica (de formato mediano).

El prototipo racial observado describe un animal eumétrico, de proporciones mediolíneas y caracterización sexual bien definida. Prácticamente todos los caracteres cumplieron el patrón racial descrito en la creación del Libro Genealógico de la raza (BOA, 2001), a excepción de la proporción mediolínea observada en el presente trabajo.

\section{CARACTERIZACIÓN REPRODUCTIVA DE LA RAZA}

El $84,4 \%$ de las corderas alcanzaron la pubertad en otoño-invierno y el 6,3\% lo hizo en la primavera siguiente $(7,9$ meses, $30,7 \mathrm{~kg}$, $65-70 \%$ peso adulto). El $55,2 \%$ de las corderas púberes mostraron fases interluteales de duración más larga de lo habitual después de la primera ovulación (ciclos largos de 4-7 semanas). Por tanto, las corderas nacidas en primavera no deberían ser cubiertas antes de que alcancen 13-18 meses de edad para evitar un posible desequilibrio entre las necesidades de crecimiento y desarrollo y su fisiología reproductiva. Por su parte, el 44,7\% de las ovejas adultas alcanzaron su primera fase luteal post-parto an- tes de finalizar la estación de primavera. La duración media del anestro post-parto en época de anestro estacionario fue de 2,5 meses. En nuestras condiciones de trabajo, la estacionalidad no sería un factor limitante en las producciones finales de los rebaños de Churra Tensina.

\section{TIPIFICACIÓN DE CANALES DE CORDERO LECHAL, TERNASCOYPASTENCO}

Las canales más ligeras presentaron el mayor rendimiento comercial $(53,8,51,4$, y $49,5 \%$, para lechal, ternasco y pastenco, respectivamente), debido quizá al menor desarrollo del tracto digestivo. Estos rendimientos fueron mayores que los descritos en otras razas españolas, probablemente porque los animales del presente ensayo no se destetaron. Como era de esperar, todas las medidas lineales realizadas sobre las canales aumentaron significativamente al incrementarse el peso de la canal, dando lugar a canales mejor conformadas $(\mathrm{O}-, \mathrm{O}+$ y R) y más engrasadas $(2,2+, 3)$, aunque se debe subrayar que las canales de lechales mostraron un grado de finalización muy aceptable. El color de la carne difirió entre tipos comerciales, mostrando un color claro las canales de lechal, rosado las de ternasco y rojo las de pastenco. No se registraron diferencias significativas en el color de la grasa de los tres tipos comerciales. Este rango de características garantiza la viabilidad comercial de los tres tipos de cordero estudiados, abriéndose una posibilidad real de crear nuevos mercados y de recuperar algunos productos tradicionales ya abandonados. Recientemente, se ha desarrollado la Marca Colectiva Lechal Tensino, cuyo reglamento ya ha sido aprobado.

\section{DIVERSIDAD GENÉTICA Y RELACIONES CON OTRAS RAZAS}

La raza Churra Tensina presentó entidad genética propia, con niveles altos de variabilidad $(\mathrm{H}=0,659)$ y bajos de consanguinidad (FIS =0,041). Se identificaron los microsatélites más informativos para la raza Chu- 
rra Tensina(BM1329, OARFCB304, MAF70, OARFCB128, OARJMP58, OARJMP29, MCM140, OARFCB226 y MAF33) con objeto de incluirlos en los test de parentesco, autentificación de productos y en el programa de conservación de la raza. Los análisis de diferenciación genética indican que no ha habido intercambio genético con otras razas del tronco churro aquí analizado. El análisis de reconstrucción filogenética agrupó a la Churra, Churra Tensina y Latxa en un cluster con una fiabilidad muy elevada. Finalmente, se han puesto a punto las diferentes metodologías laboratoriales y analíticas, que permitirán instaurar un esquema de conservación en la raza Churra Tensina, maximizando la variabilidad genética y minimizando su consanguinidad.

\section{CONSERVACIÓNDE GAMETOS YEMBRIONES}

Para garantizar la preservación de la raza Churra Tensina a largo plazo se mantienen vitrificadas todas las muestras de DNA analizadas, así como 366 dosis de semen (de 17 moruecos) y 153 embriones (de 11 machos y 35 hembras), procedentes de animales pertenecientes a los diferentes grupos

\section{BIBLIOGRAFÍA}

Álvarez-Rodríguez, J., Calvo, J.H., Revilla, R., Casasús, I. y Sanz, A. 2008. Caracterización estructural y racial de la raza ovina Churra Tensina. FEAGAS, 34: 135-143.

Avellanet, R. 2006. Conservación de recursos genéticos ovinos en la raza Xisqueta: Caracterización estructural, racial y gestión de la diversidad en programas in situ. Tesis doctoral. Universidad Autónoma de Barcelona. 282 pp.

BOA. 2001. Decreto 265/2001, de 6 de noviembre, del Gobierno de Aragón, por el que se crean los Libros Genealógicos de las razas ovinas Cartera, Churra Tensina, Maellana, Ojinegra de Teruel y caprina Moncaína.

BOE. 1997. Real Decreto 1682/1997 de 7 de noviembre, por el que se actualiza el Catálogo Oficial de Razas de Ganado de España.

Calvo, J.H., Alvarez-Rodriguez, J., Marcos- genéticos de resistencia a scrapie que existen en la actualidad.

\section{CONCLUSIONES}

Los diferentes estudios corroboran la recuperación de una raza homogénea con entidad genética propia. La viabilidad de sus productos comerciales añadida a la continuidad de las explotaciones ganaderas en las que se encuentra son aspectos que garantizan la preservación de una raza adaptada a las condiciones climáticas adversas y de determinados sistemas ganaderos ligados a la tierra, y por ende sus beneficios económicos, ambientales y sociales.

\section{AGRADECIMIENTOS}

En memoria de A. Bergua, R. Delfa y T. Fustero. Se agradece la colaboración de R. Revilla (CTA de Aragón); I. Casasús, J. Folch, E. Echegoyen, P. Sanchez, L. Cascarosa, S. Carrasco, J. Casáus, J. Ferrer, J.M. Acín y M.A. Pueyo (CITA de Aragón); R. Azón y R. Avellanet (ATURA). Financiado por los proyectos INIA RZ2004-028, RZP2004-0008 y RTA2003-031.

Carcavilla, A., Serrano, M. y Sanz, A. 2011 Genetic diversity in the Churra tensina and Churra Lebrijana endangered Spanish sheep breeds and relationship with other Churra group breeds and Spanish mouflon. Small Rum. Res., 95: 34-39.

FAO. 1996. Domestic animal diversity information system. FAO. Rome. Italy. http://www.fao.org/ dad-is/. (01/01/09).

Sanz, A. and Álvarez-Rodríguez, J. 2008. Luteal function during the first breeding and nonbreeding seasons in spring-born Churra Tensina ewe lambs. Reprod. Dom. Anim., 43: 98.

Sanz, A., Álvarez-Rodríguez, J., Cascarosa, L., Ripoll, G., Carrasco, S., Revilla, R. y Joy, M. 2008. Carcass characteristics of the commercial types of suckling lamb, light lamb and castrated lamb of Churra Tensina breed. ITEA, 104: 42-57. 